\title{
Nonlinear Control Analysis of an ICU Model for Tight Glycaemic Control
}

Levente Kovács ${ }^{1}$, Péter Szalay ${ }^{1}$, Balázs Benyó ${ }^{1}$, J. Geoffrey Chase $^{2}$

${ }^{1}$ Dept. of Control Engineering and Information Technology Budapest Univ. of Technology and Economics, Hungary

${ }^{2}$ Dept. of Mechanical Engineering, Centre for Bio-Engineering University of Canterbury, Christchurch, New Zealand 


\section{Contents}

- Introduction

$>$ Motivation

$>$ ICU models

- Investigated model

- Nonlinear synthesis

- LPV \& qALPV modeling

- Results

- Conclusions 


\section{Motivation}

\section{ICU \& TGC}

- Hypoglycaemia \& insulin resistance $\Rightarrow /$ morbidity \& mortality $^{1}$

- TGC can reduce adverse outcomes ${ }^{2}$ (and costs ${ }^{3}$ )

- Multidimensional problem (avoid hypo, variability, $\mathrm{CHO}$, etc.) ${ }^{4}$

- Repeatability problem ${ }^{5}$

- Variability in ICU patients presents ideal application field for model-based automation of insulin infusions for TGC $^{6}$

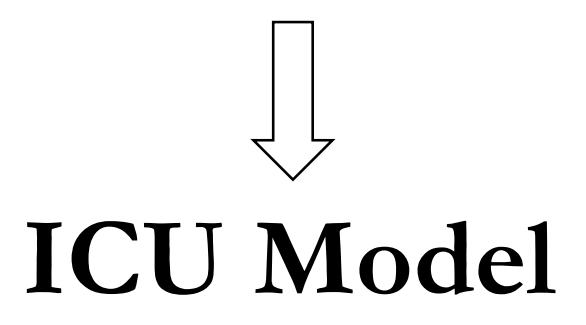

1 - SE Capes et al. (2000). Lancet, 355(9206): 773-778.

2 - J Chase et al. (2008). Critical Care, 12:R49.

3 - Van den Berghe et al. (2006). Crit Care Med, 34(3):612-616.
4 - U Pielmeier et al. (2010). UKACC Conf, 839-844.

5 - Griesdale et al. (2009) Can Med Assoc J, 180(8):821-827.

$6-\mathrm{J}$ Lin et al. (2008). CMPB, 89(2):141-152.

Contents in Introduction - Motivation - ICU models a Investigated model a Nonlinear Synthesis $a$ LPV \& qALPV modeling Results conclusions 


\section{ICU Models}

\section{Models $^{1}$}

- Minimal model: Bergman-model $(1979,1981)$

- ICU: Canterbury-model (2004, 2008, 2010)

van Herpe-model (2006)

Challenges ${ }^{1}$ :

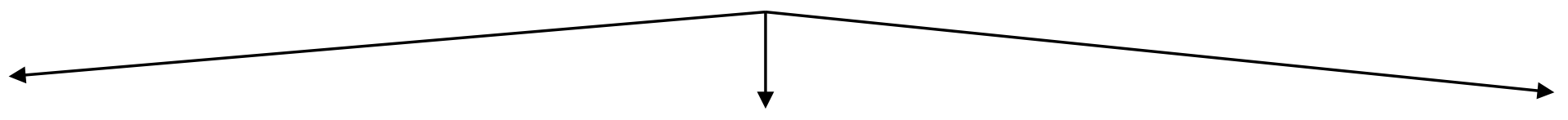

Nonlinearity

Time variance

Limited sensor capabilities

1 - L Kovacs et al. (2010). UKACC Conf. 577-582.

Contents a Introduction $\bullet$ Motivation • ICU models a Investigated model a a Nonlinear Synthesis $a$ LPV \& qALPV modeling Results a Conclusions 


\section{Canterbury-model ${ }^{1}$}

$$
\begin{aligned}
& \dot{G}(t)=-p_{G} G(t)-S_{I}(t)\left(G(t)+G_{E}\right) \frac{Q(t)}{1+\alpha_{G} Q(t)}+P(t) \\
& \dot{Q}(t)=k I(t)-k Q(t) \\
& \dot{I}(t)=-\frac{n I(t)}{1+\alpha_{I} I(t)}+\frac{u_{e X}(t)}{V}
\end{aligned}
$$

- Insulin bounded to interstitial sites

- Insulin losses to the liver and kidneys

- Saturation dynamics

- Insulin sensitivity metric 


\section{Redefined Canterbury-model ${ }^{1}$}

$$
\begin{aligned}
& \dot{G}(t)=-p_{G} G(t)-S_{I}(t) \frac{G(t) Q(t)}{1+\alpha_{G} Q(t)}+\frac{P(t)+E G P_{b}-C N S}{V_{G}} \\
& \dot{Q}(t)=k I(t)-k Q(t) \\
& \dot{I}(t)=-\frac{n I(t)}{1+\alpha_{I} I(t)}+\frac{u_{e x}(t)}{V}+\frac{u_{e n d}(t)}{V} \begin{array}{l}
\text { Actual plasma glucose } \\
\text { concentration }
\end{array} \\
& \begin{array}{l}
\text { Endogenous insulin } \\
\text { production }
\end{array} \\
& \dot{P}_{1}(t)=D(t)-d_{1} P_{1}(t) \\
& P(t)=d_{1} P_{1}(t)-\min \left\{d_{2} P_{2}(t), P_{\max }\right\}\left\{\begin{array}{l}
u_{\text {end }}(t)=k_{1} \exp \left(\frac{-k_{2} I(t)}{k_{3}}\right) \\
\begin{array}{l}
\text { Glucose absorption } \\
\text { during enteral feeding } \\
\text { (in reality are linear f.) }
\end{array}
\end{array}\right.
\end{aligned}
$$

1 - F. Suhaimi et al. (2010). UKACC Conf, 1037-1042.

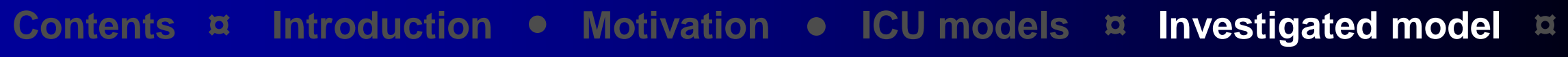
a Nonlinear Synthesis $a$ LPV \& qALPV modeling Results a Conclusions 


\section{Redefined Canterbury-model ${ }^{1}$}

MilaNi IIFAC

$\mathrm{Q}(\mathrm{t})$ is a slow variable ${ }^{1}$ :
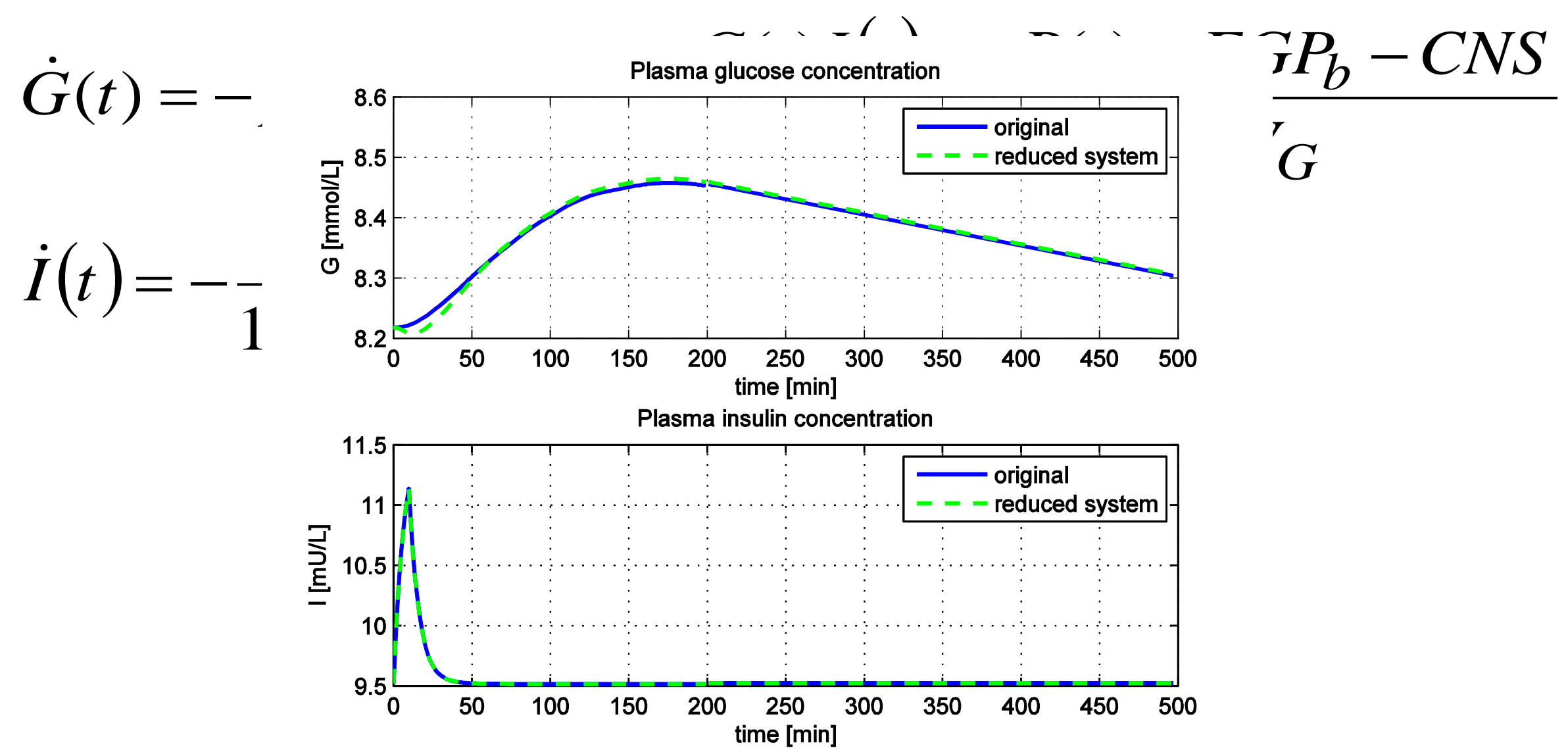

1 - E.D. Lehmann, T.A. Deutsch (1992). J Biomed Eng, 14:235-242.

Contents Introduction $\circ$ Motivation - ICU models a Investigated model a a Nonlinear Synthesis $a$ LPV \& qALPV modeling Results a Conclusions 


\section{Nonlinear analysis}

\section{Reachability}

$$
\begin{aligned}
& \dot{\varsigma}_{1}=f_{1}\left(\varsigma_{1}, \varsigma_{2}\right)+\sum_{i=1}^{m} g_{1 i}\left(\varsigma_{1}, \varsigma_{2}\right) u_{i} \\
& \dot{\zeta}_{2}=f_{2}\left(\varsigma_{2}\right) \\
& y_{i}=h_{i}\left(\varsigma_{1}, \varsigma_{2}\right)
\end{aligned}
$$

Increasing the $\Delta^{\mathrm{C}}$ distribution by its Lie-derivatives' vector fields, until the rank of the distribution increases.

\section{Observability}

$$
\begin{aligned}
& \dot{\varsigma}_{1}=f_{1}\left(\varsigma_{1}\right)+\sum_{i=1}^{m} g_{1 i}\left(\varsigma_{1}\right) u_{i} \\
& \dot{\zeta}_{2}=f_{2}\left(\varsigma_{1}, \varsigma_{2}\right)+\sum_{i=1}^{m} g_{2 i}\left(\varsigma_{1}, \varsigma_{2}\right) u_{i} \\
& y_{i}=h_{i}\left(\varsigma_{1}\right)
\end{aligned}
$$

Expending the $\mathrm{O}$ observable subspace with Lie-derivatives, until the rank of the $\mathrm{d} \Delta^{\mathrm{O}}$ co-distribution increases.

\section{Input-output linearization}

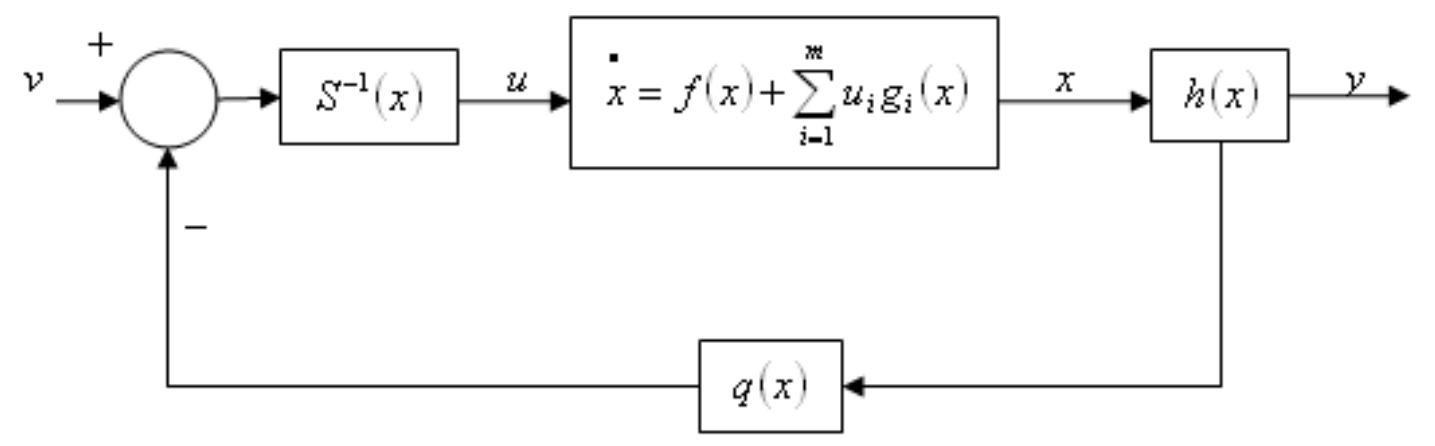




\section{Nonlinear analysis - results}

\begin{tabular}{|c|c|c|c|}
\begin{tabular}{|c|c|} 
Name of the \\
model
\end{tabular} & Dimension & Reachability & Observability \\
\hline $\begin{array}{c}\text { Canterbury- } \\
\text { model }\end{array}$ & 3 & 3 & 2 \\
G(t), Q $(\mathrm{t}), \mathrm{I}(\mathrm{t})$
\end{tabular}

plasma insulin can be estimated by knowing insulin input 


\section{Nonlinear analysis - results}

\section{Exact linearization}

$$
\begin{aligned}
& a(x)=L_{f}^{2} h(x)=\left(\left(\frac{S_{I} I \alpha_{G}}{\left(1+\alpha_{G} I\right)^{2}}-\frac{S_{I}}{1+\alpha_{G} I}\right)\left(u_{e n b}-\frac{n I}{1+\alpha_{I} I}\right)-\left(p_{G}+\frac{S_{I} I}{1+\alpha_{G} I}\right)^{2}\right) G-\left(p_{G}+\frac{S_{I} I}{1+\alpha_{G} I}\right)\left(\frac{E G B_{b}+C N S}{V_{G}}\right) \\
& b(x)=L_{g} L_{f}^{1} h(x)=\frac{-S_{I} G}{\left(1+\alpha_{G} I\right)^{2} V_{I}} \\
& u_{e x}=\frac{1}{b(x)}(u-\alpha(x))
\end{aligned}
$$

$$
\left.\stackrel{v}{u=\frac{1}{b(x)}(v-\alpha(x))} \stackrel{u}{\longrightarrow} \begin{array}{l}
\dot{x}=f(x)+g(x) u \\
y=h(x)
\end{array}\right) \stackrel{y}{\longrightarrow}
$$




\section{LPV Modeling}

Nonlinear model based design technique (extension of LTI systems) ${ }^{1,2}$

$$
\begin{aligned}
& \dot{x}(t)=A(\rho(t)) x(t)+B(\rho(t)) u(t) \\
& y(t)=C(\rho(t)) x(t)+D(\rho(t)) u(t)
\end{aligned}
$$

$\varrho(t)$ should be known

by measurement or

computation

2 well-known techniques:

- affine type: a part of the $\varrho(t)$ are equal with the $x(t)$ states

- polytope type: the validity of the model is caught inside a polytope region $\Longrightarrow$ linear combination of linear models

$$
\Sigma(t) \subset\left\{\Sigma_{1}, \ldots, \Sigma_{2}\right\}=\left\{\sum_{i=1}^{j} \alpha_{i} \Sigma_{i}: \alpha_{i} \geq 0, \sum_{i=1}^{j} \alpha_{i}=1\right\} \quad \Sigma_{i}=\left[\begin{array}{cc}
A_{i} & B_{i} \\
C_{i} & D_{i}
\end{array}\right]
$$

1 - F Wu et al. (2000). Int J Control, 73(12): 1104-1114.

2 - W Tan (1997). Applications of Linear Parameter-Varying Control Theory. MSc. thesis, Berkeley.

Contents a Introduction $\circ$ Motivation $\bigcirc$ ICU models a Investigated model a a Nonlinear Synthesis LPV \& qALPV modeling a Results conclusions 


\section{qALPV}

Affine dependency:

$$
\begin{aligned}
& A(\rho)=A_{0}+\rho_{1} A_{1}+\ldots+\rho_{N} A_{N} \\
& B(\rho)=B_{0}+\rho_{1} B_{1}+\ldots+\rho_{N} B_{N} \\
& C(\rho)=C_{0}+\rho_{1} C_{1}+\ldots+\rho_{N} C_{N} \\
& D(\rho)=D_{0}+\rho_{1} D_{1}+\ldots+\rho_{N} D_{N} \\
& \Sigma(t)=\left\{\Sigma_{0}+\sum_{i=1}^{N} \rho_{i} \Sigma_{i}: \rho_{i} \in\left[\underline{\rho}_{i} \bar{\rho}_{i}\right], \dot{\rho}_{i} \in\left[\dot{\rho}_{i} \dot{\bar{\rho}}_{i}\right]\right\} \quad \Sigma_{i}=\left[\begin{array}{ll}
A_{i} & B_{i} \\
C_{i} & D_{i}
\end{array}\right]
\end{aligned}
$$

Three possibilities:

- Jacobi linearization

- state transformation

- function substitution 


\section{qALPV $\rightarrow$ Results}

Canterbury-model

$$
\begin{gathered}
\dot{G}(t)=-p_{G} G(t)-S_{I}(t) \frac{G(t) I(t)}{1+\alpha_{G} I(t)}+\frac{P(t)+E G P_{b}-C N S}{V_{G}} \\
\dot{I}(t)=-\frac{n I(t)}{1+\alpha_{I} I(t)}+\frac{u_{e x}(t)}{V}+\frac{u_{e n d}(t)}{V} \\
\rho(t)=\left[\begin{array}{c}
\rho_{1}(t) \\
\rho_{2}(t)
\end{array}\right]=\left[\begin{array}{c}
\left.S_{I}(t) \frac{I(t)}{1+\alpha_{G} I(t)}\right] \\
\frac{1}{1+\alpha_{I} I(t)}
\end{array}\right] \text { Can be calculated }
\end{gathered}
$$




\section{qALPV $\rightarrow$ Results}

Canterbury-model

$$
\begin{gathered}
\dot{G}(t)=-p_{G} G(t)-S_{I}(t) \frac{G(t) I(t)}{1+\alpha_{G} I(t)}+\frac{P(t)+E G P_{b}-C N S}{V_{G}} \\
\dot{I}(t)=-\frac{n I(t)}{1+\alpha_{I} I(t)}+\frac{u_{e x}(t)}{V}+\frac{u_{e n d}(t)}{V} \\
A(\rho(t))=A_{0}+A_{1} \rho_{1}(t)+A_{2} \rho_{2}(t)= \\
=\left[\begin{array}{cc}
p_{G} & 0 \\
0 & 0
\end{array}\right]+\left[\begin{array}{cc}
-1 & 0 \\
0 & 0
\end{array}\right] \rho_{1}(t)+\left[\begin{array}{cc}
0 & 0 \\
0 & -n
\end{array}\right] \rho_{2}(t) \\
B_{1}=\left[\begin{array}{l}
0 \\
\frac{1}{V}
\end{array}\right], B_{2}=\left[\begin{array}{l}
1 \\
0
\end{array}\right], C=\left[\begin{array}{ll}
1 & 0 \\
0 & 1
\end{array}\right] .
\end{gathered}
$$




\section{qALPV $\rightarrow$ Results}

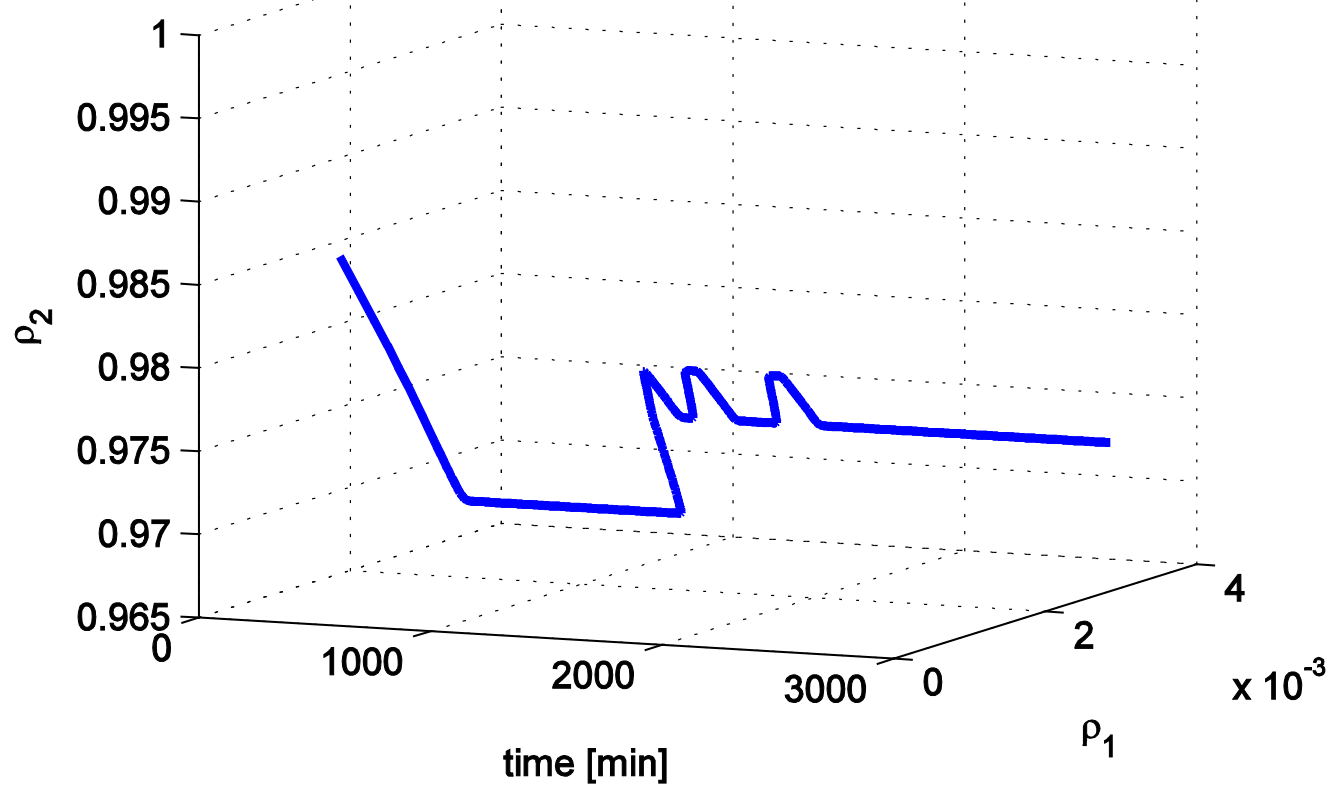

\begin{tabular}{|c|c|c|c|}
\hline \multicolumn{2}{|c|}{ Scheduling parameter } & By measurement & Theoretical bound \\
\hline \multirow{2}{*}{$\rho_{1}$} & $\underline{\rho}_{1}$ & 0.0019 & $0($ if $\mathrm{I} \rightarrow 0)$ \\
\cline { 2 - 4 } & $\bar{\rho}_{1}$ & 0.0034 & $0.0146($ if I $\rightarrow \infty)$ \\
\hline \multirow{2}{*}{$\rho_{2}$} & $\underline{\rho}_{2}$ & 0.9672 & $0($ if I $\rightarrow \infty)$ \\
\cline { 2 - 4 } & $\bar{\rho}_{2}$ & 0.9841 & $1(\mathrm{I} \rightarrow 0)$ \\
\hline
\end{tabular}

Contents is Introduction $\circ$ Motivation $\circ$ ICU models in Investigated model to Nonlinear Synthesis LPV \& qALPV modeling a Results Conclusions 


\section{qALPV vs. nonlinear system}

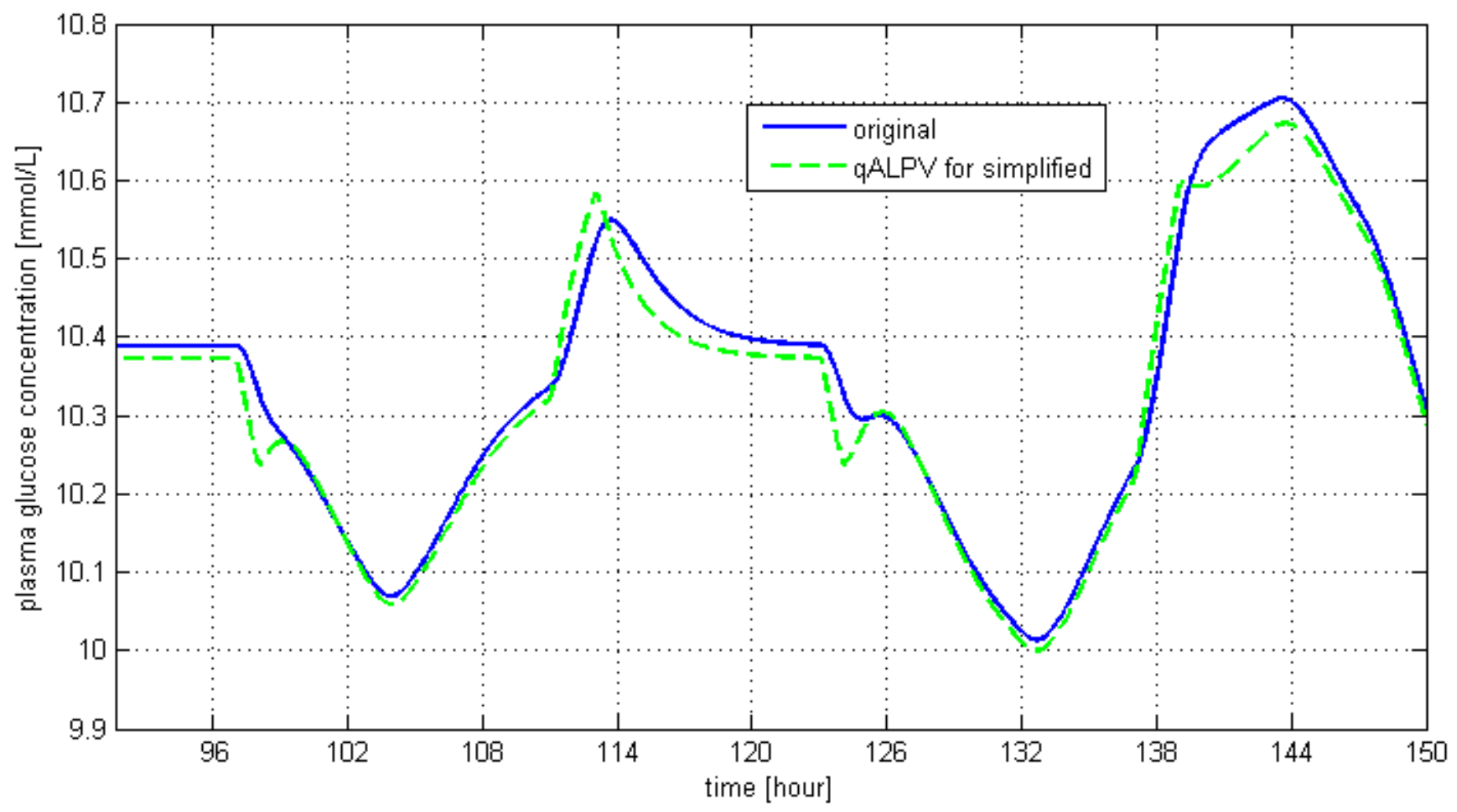

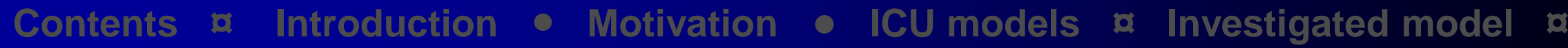
o Nonlinear Synthesis a LPV \& qALPV modeling of Results conclusions 


\section{Conclusions}

- Nonlinear control analysis roadmap of a frequently used ICU model

- Nonlinear analysis

- qALPV investigation and formulation

- Further work:

$>$ robust control method $\left(\mathrm{H}_{\infty}\right)+$ new Canterbury-model (2010)

$>$ simulations of different real-life scenarios (per patient management) 


\section{Thank you for your attention!}

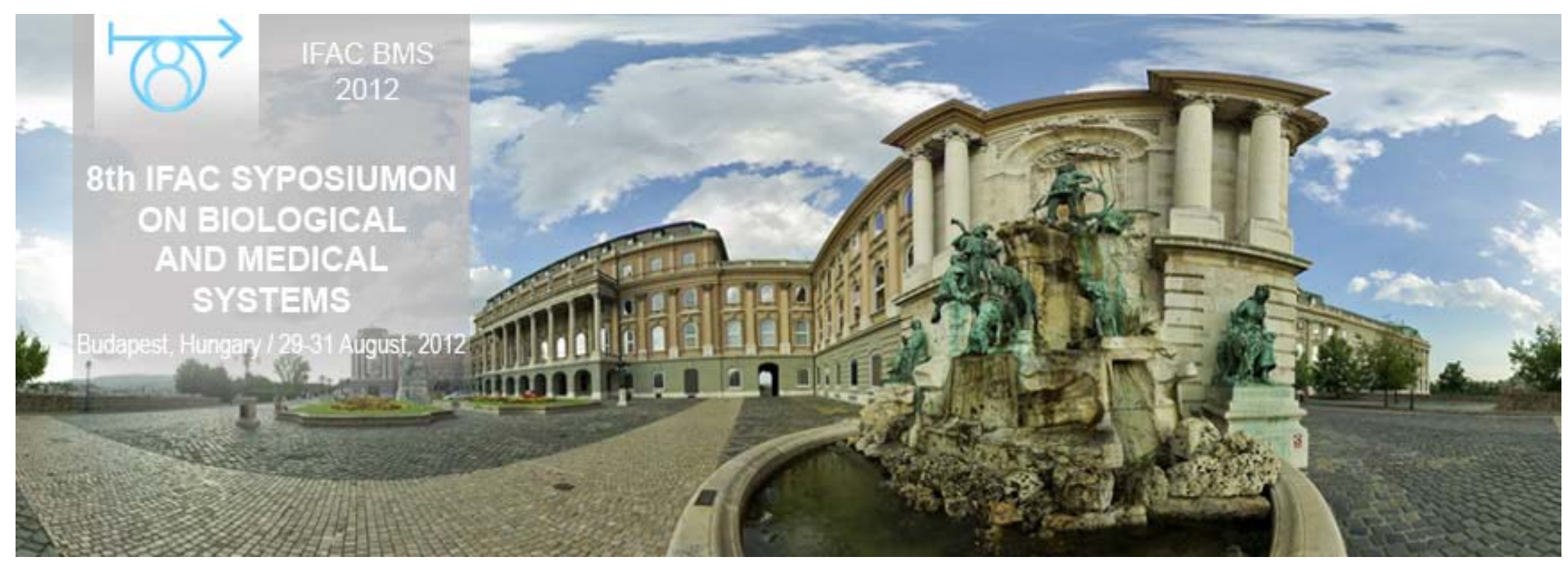

8th IFAC Symposium on Biological and Medical Systems Budapest, Hungary / 29-31 August, 2012 